\title{
Rolic $^{\circledR}$ LCMO Photo Alignment Technology: Mechanism and Application to Large LCD Panels
}

\author{
Mohammed Ibn-Elhaj ${ }^{1}$, Sabrina Chappellet and Frédéric Lincker \\ Rolic Technologies Ltd. CH-4123 Allschwil, Switzerland \\ 1mohammed.ibn-elhaj@rolic.ch
}

\begin{abstract}
Keywords: LCMO (Light Controlled Molecular Orientation); Photo-alignment; Cross-linking and stability; Displays; VA-LCDs; 3D Patterned-Retarders; Optical Security Devices; Low energy consumption
\end{abstract}

\begin{abstract}
Rolic ${ }^{\circledR}$ Light Controlled Molecular Orientation (LCMO) technology is the basis for todays advanced mass production technologies for large LCD-TV panel, high-resolution 3D patterned-retarders and high-resolution optical security devices. This fundamental technology allows an easy achievement of high resolution azimuthal LC-director patterns with defined bias angles, from homogeneous planar to homeotropic orientation, depending on the target application [1-7]. In addition to the control of bias angles, LCD panel manufacturers require alignment layers with a wide range of optimized properties. Thin alignment films must be easily coatable and should have high photosensitivity in order to achieve fast processing. The photoalignment film should also have high stability and good electrical properties such as Voltage Holding Ratio (VHR), Residual DC (RDC) and image sticking.

Because of in-situ photo crosslinking during processing [1, 2], our proprietary LCMO photoalignment technology is shown to be thermally and optically stable. Last years, enormous progress has been made in the development of advanced materials that meet all requirements for mass production of large-area flat panel displays. LCMO-VA technology, for vertical alignment LCDs, is the basis for the state of the art $U^{2}$ A production technology recently used in the manufacturing of advanced new generation LCD-TV panel displays with reduced production costs and low energy consumption $[8,9]$. LCMO-VA mechanism and performances of state of the art materials will be discussed.
\end{abstract}

\section{Introduction}

Homogeneous and stable alignment of liquid crystals (LCs) with well defined bias tilt angles over large areas is a prerequisite for large-scale manufacturing of liquid crystal displays (LCDs) as well as other LC based devices such as optically patterned retarders for 3D and anti-counterfeiting. Until recently conventional alignment methods such as the rubbing of polyimide layers or geometrical patterning (such as topologies or protrusion) via photo-litoghraphic processing were the methods of choise for large-scale production of LCDs. The rubbing of polyimide generates dust, static electricity and mechanical damage which lower the yield and performances of LCDs. Also, microtopologies reduce LCD performances such as light transmission and response time [8]. Morevover, the obtention of multi-domains with different LC director orientation, to improve viewing angles, requires complicated and costly processing and reduces yield. Therefore, more reliable production methods are needed to reduce costs of large-area flat panel displays and improve their performances.

One of the most attractive alternative is the use of photo-alignment or optical alignment to generate anisotropic alignment films. In the 1980's first attempts were made to induce molecular reorientation in guest host materials, comprising mainly azo guest molecules, by exposing to UV light [10-12]. In the early 1990's two research groups showed polarised UV exposure to induce an in-plane anisotropy in a polymer doped with an azo dye [13] and in polyvinylcinnamate (PVCin) polymers based on photo-crosslinkable cinnamate chromophores [1]. Meanwhile, other materials and photo-alignment processes have been proposed, such as photo-degradation with deep UV light[15]. Therefore, mainly three fundamentally different aligning strategies by linearly polarised 
light were pursued, namely (i) optically induced cis-trans isomerisation of single-chromophores, (ii) photo-degradation or photo-rubbing of polyimide layers and (iii) photo-alignment with crosslinkable materials.

Photo-alignment process with cis-trans isomerisation is based on reorientation of single chromophores and generally suffers from stability; for example, azo dyes doped in polymers, such as polyimide [13] and polyvinyl alcohol [14] give arise to instable alignment. The photo-rubbing or photo-degradation process is attributed to directional bleaching or decomposition of polyimide layers by linear polarized light [16] via chains broken to form free radicals, which then take part in oxidation reactions. Photosensitivity is an important parameter since high UV doses and, therefore, long exposure times are usually required to achieve photoalignment. Moreover, decomposed moieties will negatively affect LCD performances. So far, LCDs had not been produced commercially with cis-trans isomerisation and photo-degradation photo-alignment technologies.

Because of in-situ photo crosslinking during processing [1, 2], our proprietary LCMO photoalignment technology is inherently thermally and optically stable. This renders LCMO the fundamental photo-alignment technology for many displays and non-displays applications. In addition to LCMO-VA technology for VA-LCDs that will discuss in this paper, other LCMO technologies also break through commercialisation. LCMO-3D for manufacturing of highresolution patterned-retarders for 3D displays on large glass and recently on plastic via roll-to-roll coating. Another achievement in non-display applications is customer specific manufacturing of first level (such as SECUNOTE ${ }^{\text {TM }}$ Diamond) as well as second level (such as SECUNOTE TM Oyster) optical security devices for governmental fiscal and identity documents.

LCMO-VA Photo-aligned Multi-domain VA LCDs have been demonstrated by Rolic already in 1999 [20]. Commercial penetration of the technology have been achieved few years ago thanks to the enormous development progress done at Rolic last years together with an efficient collaboration with panel manufacturer [8,9]. Beside processing speed, optical and electrical performances, the main problem that was hindering commercial exploitation of photoaligned films is their limited alignment stability. Our newly developed LCMO-VA photoalignment technology for multi-domain VA-LCDs, allows high speed generation of homogeneous and stable bias tilt angles without fluctuations over large areas.

State of the Art LCMO-VA Materials. It is obvious that photo-sensitivity, photo-reactions involved as well as LCD performances depend on chemical structure of the material. Fig. 1 shows base molecular structure of typical state of the art Rolic vertical alignment (VA) material used in mass production of VA-LCDs.

Fig. 1. Base molecular structure of typical Rolic vertical alignment (VA) material used in mass production of VALCDs; ROP-VA. Polyamic acid and/or polyimide comprising a side chain cinnamic acid photoreactive group linked on one side, via a linker $(\mathrm{Lg})$, to an end group $(\mathrm{Eg})$ and on the other side to an amine based polymerisable group $(\mathrm{Pg})$, via a spacer (Sg). In the typical LCMO-VA material, used in this study, the end group Eg is based on fluorinated moiety $-\mathrm{Cn}-\mathrm{F} 2 \mathrm{n}+1$.

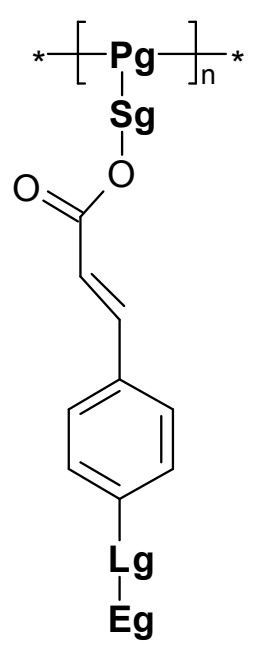


Based on Rolic long-term expertise and design capabilities, the different groups can be fine tuned to control alignment, alignment homogeneity, pretilt and their stability as well as other required electrical- and electro-optical performances such as Voltage Holding Ratio (VHR), Residual DC (RDC) and image sticking. In this paper we will discuss the intrinsic photocrosslinking mechanism of LCMO-VA and its role in enhancing stability of ROP-VA alignment layers.

\section{Photo-Crossling with Linearly Polarised UV Light (LCMO-VA)}

The photochemical reactions which occur upon exposure of cinnamate polymers with unpolarised light [17] have been analytically investigated for some classical photopolymers such as PVCin. It was clearly established the existence of cyclic products in unpolarised-UV irradiated PVcin polymer films. Cycloaddition should not to be the only photoprocess occurring in PVcin, especially in solutions. In the polymer film of PVCin, however, it was found that isomerisation plays only a minor role and does not seriously interfere with cyclization or any other bimolecular photoprocess [17]. In this section we investigate photo-reactions of typical ROP-VA material exposed to linearly polarised UV (LPUV) light. Our investigation procedure is based on one hand on combination of several analytical tools and on other hand on use of model compounds to probe photochemical reaction mechanisms and elucidate specific signatures of the possible photoproducts. In the following, we will limit our discussions to some results based on UV-vis, NMR and LC/MS spectroscopies.

Ethyl cinnamate (EtCin), which is the basic photo-reactive group of ROP-VA, have been used as model compounds to clarify the difference between trans-cis isomerisation and cycloaddition. In addition to Trans isomer (Trans-1), we also synthesized the Cis isomer (Cis-1) and the cyclodimer (Dimer-1), indicated in figure 2.

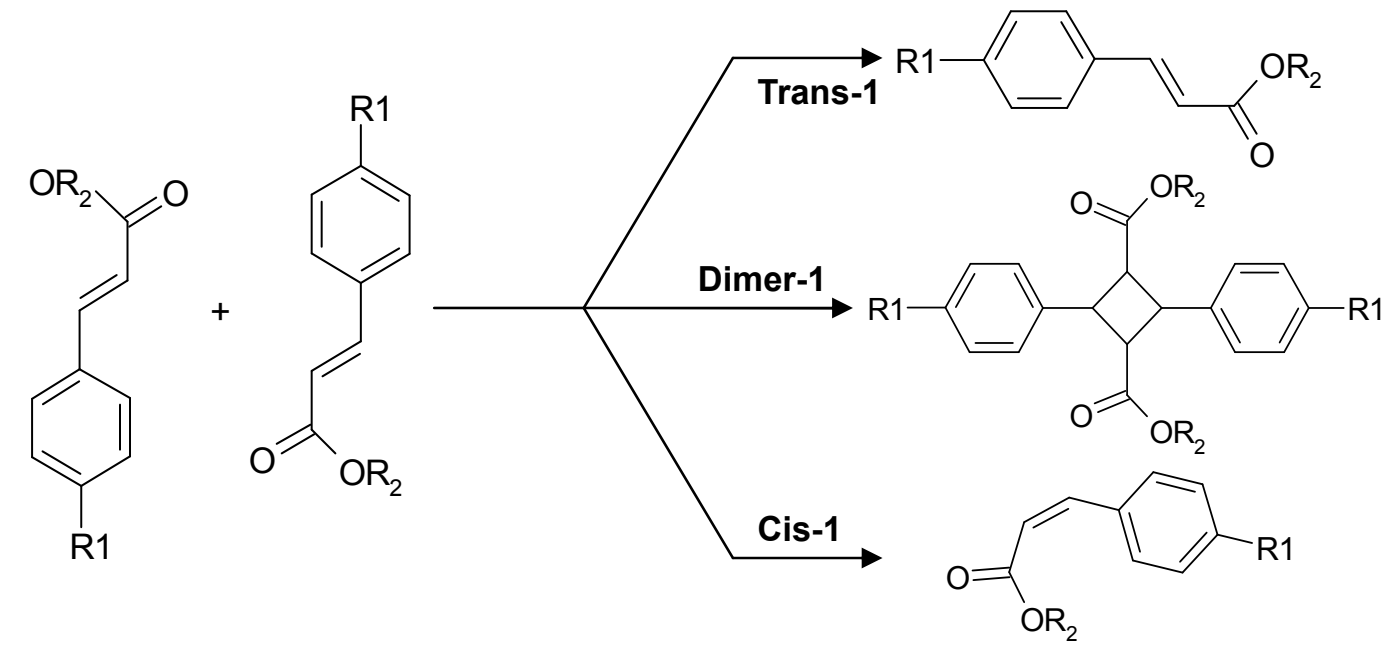

Fig. 2. Main possible photoreaction products after exposure to UV light: no-reaction (Trans-1), cis-isomerisation (Cis-1) and cyclo-addition or dimerisation (Dimer-1); with $\mathrm{R} 1=\mathrm{OH}$ and $\mathrm{R} 2=\mathrm{C}_{2} \mathrm{H}_{5}$.

The model compounds Trans-1, Cis-1 and Dimer-1 proved chromatographically homogenous and displayed spectral characteristics consistent with its assigned structure. Indeed, the pure model compounds Trans-1, Cis-1 and Dimer-1 were characterized by ${ }^{1} \mathrm{H}$ NMR spectroscopy in DMSO-D6 (deuterated dimethylsulfoxyde). Fig. 3a clearly shows the differences observed between the three model compounds. The structures of the Trans- 1 and Cis- 1 were unambiguously confirmed by the presence of characteristic signals and coupling constants of the vinylic double bond protons at $\delta 6.38$ and $7.55 \mathrm{ppm}$ with ${ }^{3} \mathrm{~J}_{\text {trans }}=15.9 \mathrm{~Hz}$ for Trans- 1 and 5.76 and $6.85 \mathrm{ppm}$ with ${ }^{3} \mathrm{~J}_{\text {cis }}=12.9 \mathrm{~Hz}$ for Cis-1. The structure of Dimer-1 was confirmed by the extinction of the signals corresponding to 
vinylic protons and the presence of two characteristic dd due to the cyclobutane ring formation. The hydrogen chemical shifts correspond to an AA'XX' pattern, HA and HX having shifts of 3.76 and $4.20 \mathrm{ppm}$ respectively.with coupling constants ${ }^{3} \mathrm{~J}$ of $7.5 \mathrm{~Hz}$ and $10.5 \mathrm{~Hz}$.. These results are in accordance with NMR data table results [18].

Fig. 3a. NMR Signatures of EtCin model compounds Trans-1, Cis-1 and Dimer-1

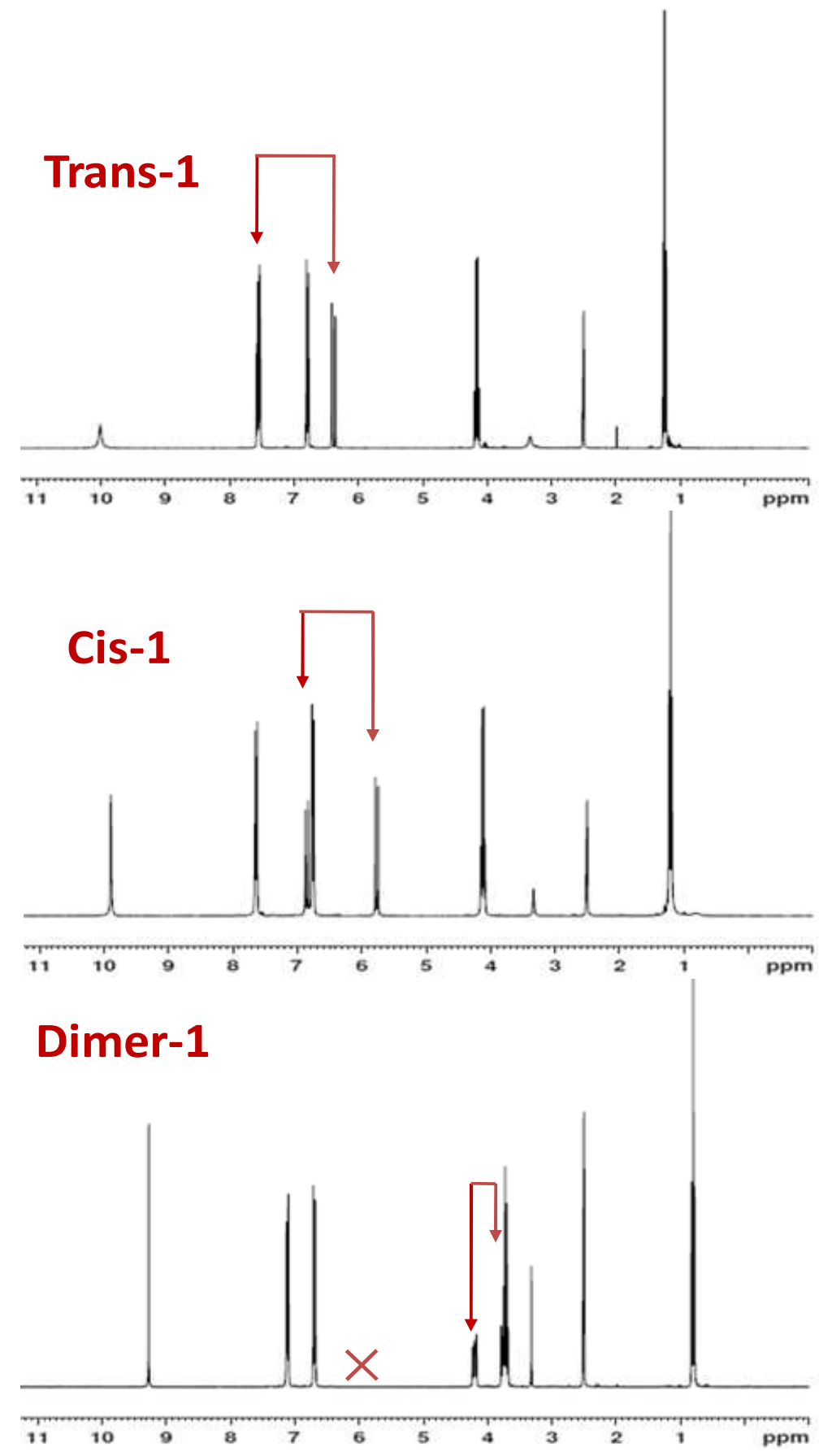

The above method was then applied to ROP-VA based chromophores. We have found that, the position and the constant coupling of the vinylic double bond protons lead to distinguish the cis from trans one; i.e. two doublets at 6.38 and $7.55 \mathrm{ppm}$ with $3 \mathrm{Jtrans}=15.9 \mathrm{~Hz}$ for the transconfiguration and two doublets at 5.77 and $6.85 \mathrm{ppm}$ with $3 \mathrm{Jcis}=12.9 \mathrm{~Hz}$ for the cis-configuration. However, due to the efficient cross-linking with LPUV exposure, ROP-VA polymer films are insoluble in common solvents including DMSO-D6 and therefore could not be analysed with the above ${ }^{1} \mathrm{H}$ NMR method.

The comparison of isotropic UV-vis absoption spectra of Trans-1, Cis-1 and Dimer-1, in acetonitrile solution clearly indicates that the positions of the absorption bands, as well as their intensity, are strongly affected by the presence and the configuration of the vinylic double bond. (Fig. 3b). As a general trend, absorption spectra of cinnamate containing molecules, i.e. Trans-1 and 
Cis-1, exhibit two characteristic absorption peaks in two distinctive ranges (Fig. 3b). The first is located in UV part of the spectrum below $230 \mathrm{~nm}$, whereas the second one appearing as broad and intense peak in the limit of the UV range $c a .290 \mathrm{~nm}$. The origin of this second absorption band for Trans- 1 and Cis- 1 was attributed to the $\pi-\pi^{*}$ transition of the vinylic double bond and the aromatic ring being in conjugation. However, the UV absorption spectrum of Dimer-1 shows a disappearance of the signal corresponding to cinnamate absorption and the presence of a strong absorption at $c a$. $195 \mathrm{~nm}$, due to the cyclobutane photo-product. The clear differences between cinnamate-based model compounds and cyclobutane adduct confirm the efficiency of the UV-vis absorption spectroscopy for highliting the [2+2]-cyclo-addition process.

Fig. 3b. Isotropic absorption spectra of cinnamate model compounds in acetonitrile solutions $\left(\mathrm{C}=5.6 \times 10^{-6}\right.$ mol. $\left.L^{-1}\right) ; \quad$ line (Trans-1), open squares (Cis-1) and open triangles (Dimer-1).

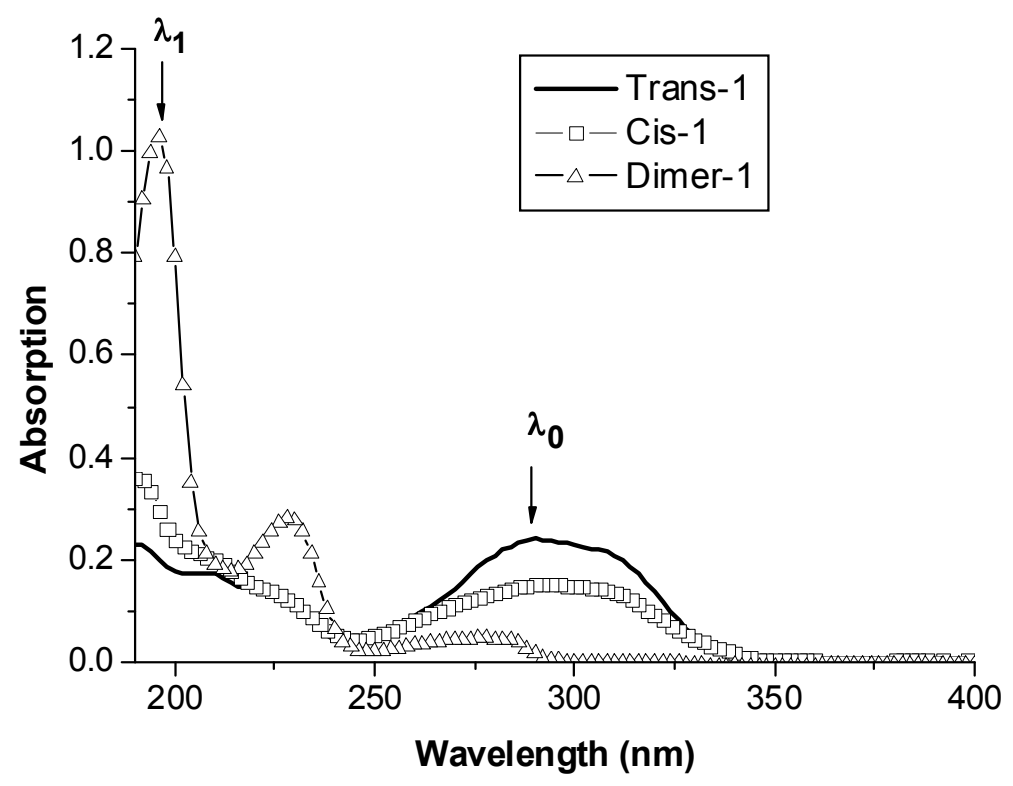

Detection of cyclobutane products in ROP-VA thin films exposed to LPUV light. Thin films of typical ROP-VA (ca. $90 \mathrm{~nm}$ ) were spin-coated on glass substrate, thermally annealed and exposed to LPUV (UVB) at different doses between about 20 and $3000 \mathrm{~mJ}$. We have found that exposed films are insoluble in common solvents, starting from low exposure doses, which is already a clear indication that cross-linking occurs in our ROP-VA materials. For a detailed analysis of reactions products to check existence or not of [2+2] cyclo-addition dimers, we used a fine tuned LC/MS (Liquid Chromatography coupled with Electrospray Mass Spectroscopy) method. The above exposed films are scratched from the substrates and hydrolysed in an acidic medium. The different solutions, corresponding to different exposure doses, were stirred at reflux until total dissolution of the scratched solid material. In principle, if [2+2] cyclo-addition occurs during LPUV exposure, the solutions should contain cyclobutane compounds (dimers). Fig. 4 shows some possible dimers that might result from hydrolysis in acidic medium (degradation) of LPUV exposed ROP-VA materials. 

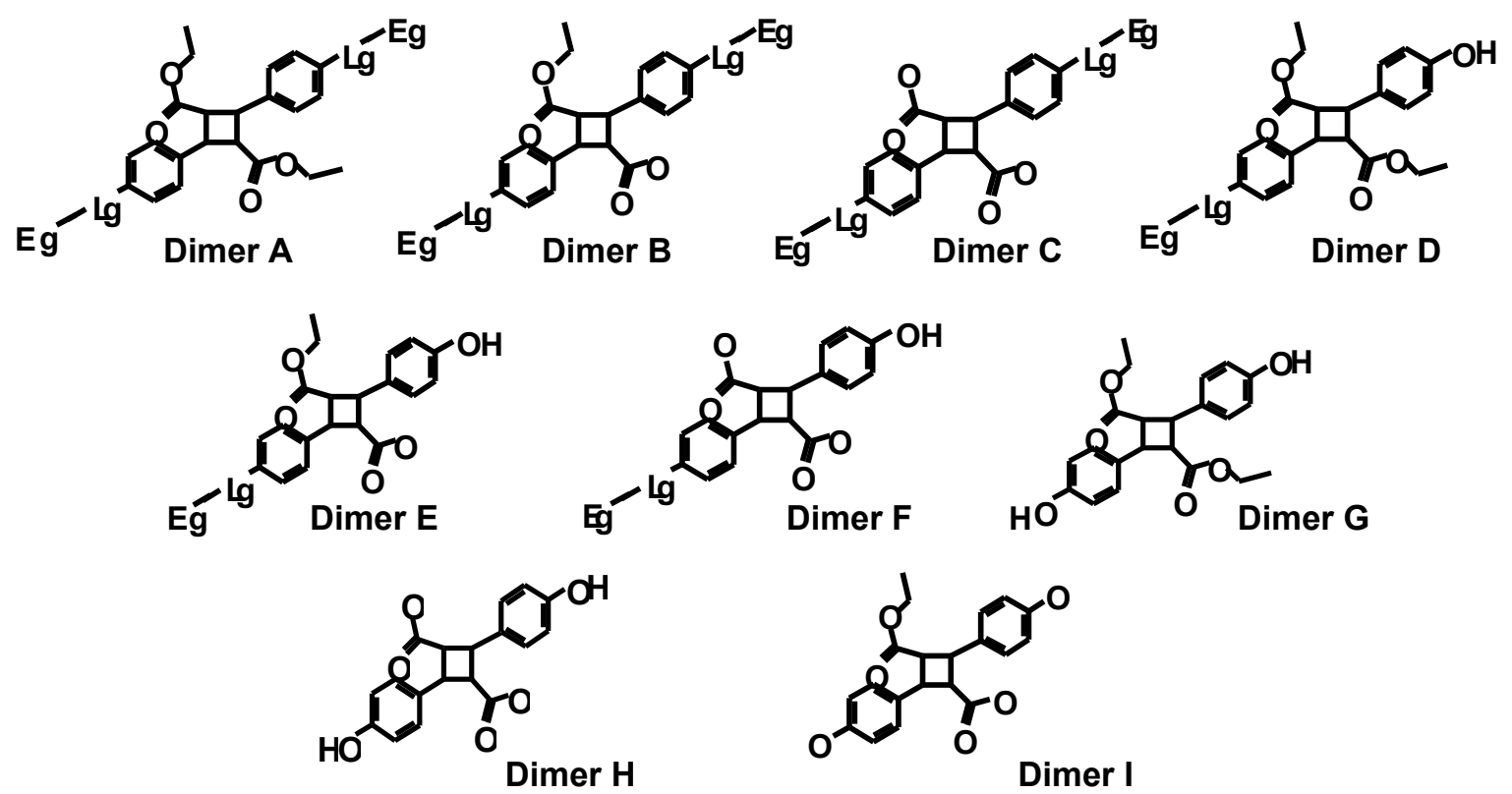

Fig. 4. Some possible dimers that can result from acidic degradation of LPUV exposed thin film of typical ROP-VA.

Table 1. Retension times in minutes and mass of some reference dimers.

\begin{tabular}{|c|c|c|}
\hline Compound & LC $(\mathbf{m i n})$ & $\mathbf{m} / \mathbf{z}(\mathbf{D A})$ \\
\hline Dimer G & $9.89 ; 10.32$ & 384.8 \\
\hline Dimer E & $17.42 ; 17.61$ & 586.6 \\
\hline Dimer D & $20.797 ; 21.084 ; 21.439$ & 614.6 \\
\hline Dimer B & 28.858 & 816.4 \\
\hline Dimer A & $32.23 ; 32.63 ; 32.84 ; 33.62$ & 844.4 \\
\hline
\end{tabular}

Some chemically stable dimers such as A, G and $\mathrm{H}$ were synthesized and isolated. These dimers have been used as reference compounds; Table 1 shows retention time and corresponding mass experimentally obtained with LC/MS which of course correspond to the theoritical values. After that, the solutions of hydrolysed LPUV exposed ROP-VA have been analysed regarding cyclobutane dimers in the hydrolysate with the same method as for reference compounds. The obtained results show the existence of peaks corresponding to retention time and mass of dimer $\mathrm{G}$ and dimer D at $3000 \mathrm{~mJ}, 200 \mathrm{~mJ}$ as well as at low LPUV doses of about $20 \mathrm{~mJ}$. The result confirms that dimerisation of ROP-VA has occurred during LPUV exposure already at low LPUV doses.

\section{Anisotropic Optical Properties Induced in Thin Films of ROP-VA exposed to LPUV}

In the previous section, we have shown the detection of [2+2] cyclo-addition dimers and hence cross-linking and photo-polymerisation in thin films of ROP-VA exposed to LPUV. In this section we investigate occurence of anisotropy in such LPUV exposed ROP-VA polymer films. Fig. 5a shows UV-absorption spectra of $150 \mathrm{~nm}$ thick, spin coated ROP-VA films on a quartz substrate. The spectra were recorded before $\left(0 \mathrm{~mJ} / \mathrm{cm}^{2}\right)$ and after exposure to polarised UVB light at different doses of 22,200 and $1000 \mathrm{~mJ} / \mathrm{cm}^{2}$. 


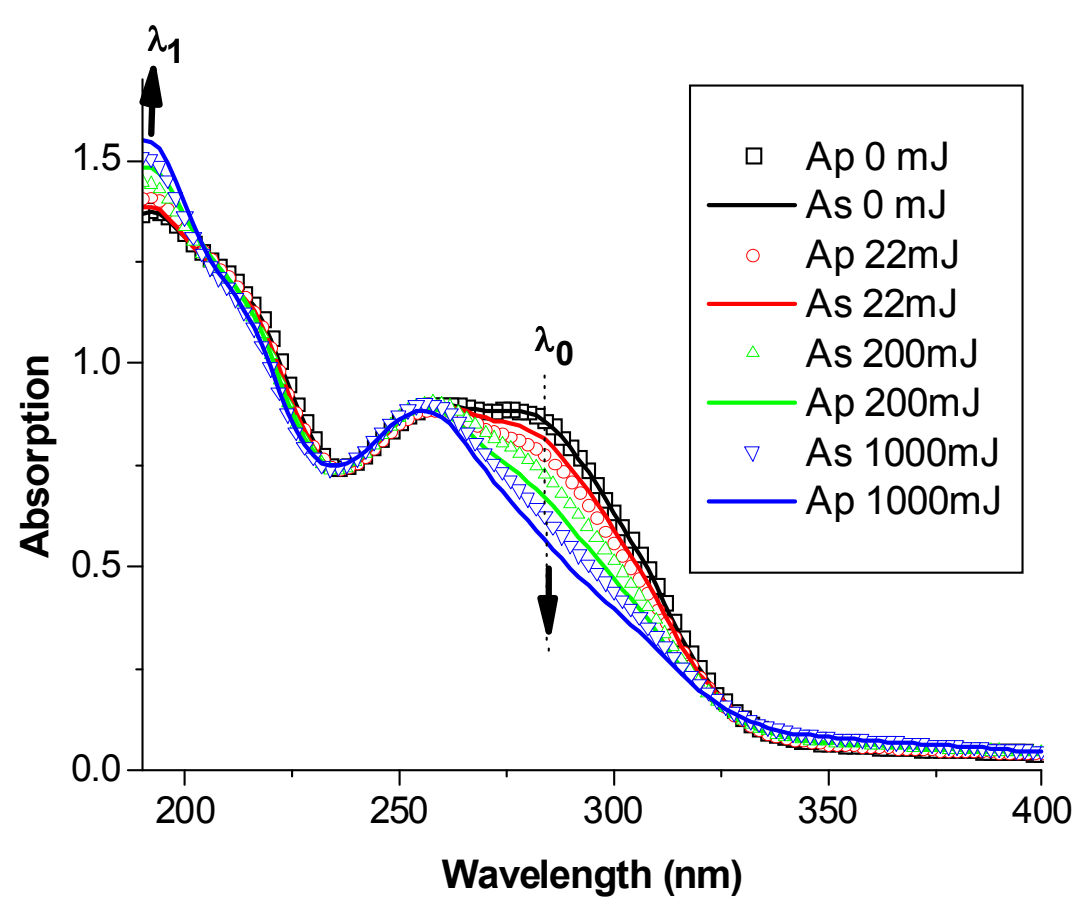

Fig. 5a. Anisotropic UV-absorption spectra of ROP-VA film prior to linearly polarised UV-B exposure $\left(0 \mathrm{~mJ} / \mathrm{cm}^{2}\right)$, as well as after exposure to LPUV doses of 22,200 and $1000 \mathrm{~mJ} / \mathrm{cm}^{2}$. Ap is the absorption with polarised light parallel to LPUV direction and As is the absorption with polarised light perpendicular to LPUV direction.

Unexposed ROP-VA films $\left(0 \mathrm{~mJ} / \mathrm{cm}^{2}\right)$ exhibit a large and strong absoption at $\lambda_{0}=284 \mathrm{~nm}$, corresponding to the $\pi-\pi^{*}$ transition of the vinylic double bond and the aromatic ring being in conjugation. With processing photopolymerisation, double-bonds are consumed for the dimerisation process and this peak decreases. Simultaneously the second short-wavelength absorption peak at $\lambda_{1}=192 \mathrm{~nm}$, corresponding to cyclobutane photo-products absorption, increases. This shift $\lambda_{0} \rightarrow \lambda_{1}$ results from the reduction of $\pi$-conjugation of the higher conjugated cinnamic side chains into cyclobutane derivatives upon cross-linking, in line with our UV-vis absorption results on model compounds and with LC/MS results which demonstrate the occurrence of dimers in thin films of ROP-VA exposed to LPUV. It is intesreting to note that the peak position of $\lambda_{1}$ at $192 \mathrm{~nm}$ (Fig. 5a) instead of $195 \mathrm{~nm}$ for Dimer 1 (Fig. 3b) is related to the molecular structure of the resulting dimers. For example, as shown in Fig. 5b, the UV absorption spectra of Dimer A is shifted to lower wavelengths compared to that of Dimer G.

To detect possible optical anisotropies in ROP-VA films exposed to LPUV light, we investigated the dichroic UV-absorption $\Delta \mathrm{A}=\left(\mathrm{A}_{\mathrm{p}}-\mathrm{A}_{\mathrm{s}}\right)$ before and after LPUV exposure. Fig. 5c shows the results calculated from the spectra of Fig. 5a. As expected, the ROP-VA coated substrate without exposure to LPUV exhibits virtually no dichroism. However, after exposure to LPUV, the inially isotropic absorption of the non-irradiated film becomes strongly negatively dichroic at $\lambda_{0} \cong 284 \mathrm{~nm}$ in the range of cinnamate absorption and positively dichroic at $\lambda_{1} \cong 192 \mathrm{~nm}$, i.e. in the photoinduced cyclobutane derivatives absorption range. 
Fig. 5b. Isotropic absorption spectra of thin films of typical dimers model compounds; open squares (Dimer A) and closed squares (Dimer G).
Fig. 5c. Dichroism $\Delta \mathrm{A}=$ $\left(A_{p}-A_{s}\right)$ of the UVabsorption of a $150 \mathrm{~nm}$ thin ROP-VA film exposed to linearly polarised UV (LPUV) light. Line (before LPUV exposure; 0 $\left.\mathrm{mJ} / \mathrm{cm}^{2}\right), \quad$ squares $\quad(22$ $\left.\mathrm{mJ} / \mathrm{cm}^{2}\right)$, circles $\quad(200$ $\mathrm{m} / \mathrm{cm}^{2}$ ) and triangles (1000 $\mathrm{mJ} / \mathrm{cm}^{2}$ LPUV exposure).

Fig. 5d. Variation of dichroism $\Delta \mathrm{A}=\left(\mathrm{A}_{\mathrm{p}}-\mathrm{A}_{\mathrm{s}}\right)$ of the UV-absorption ROP-VA films; Squares $(\triangle \mathrm{A}$ calculated from the peak at $284 \mathrm{~nm}$ ) and Circles ( $\Delta \mathrm{A}$ calculated from the peak at $192 \mathrm{~nm})$.
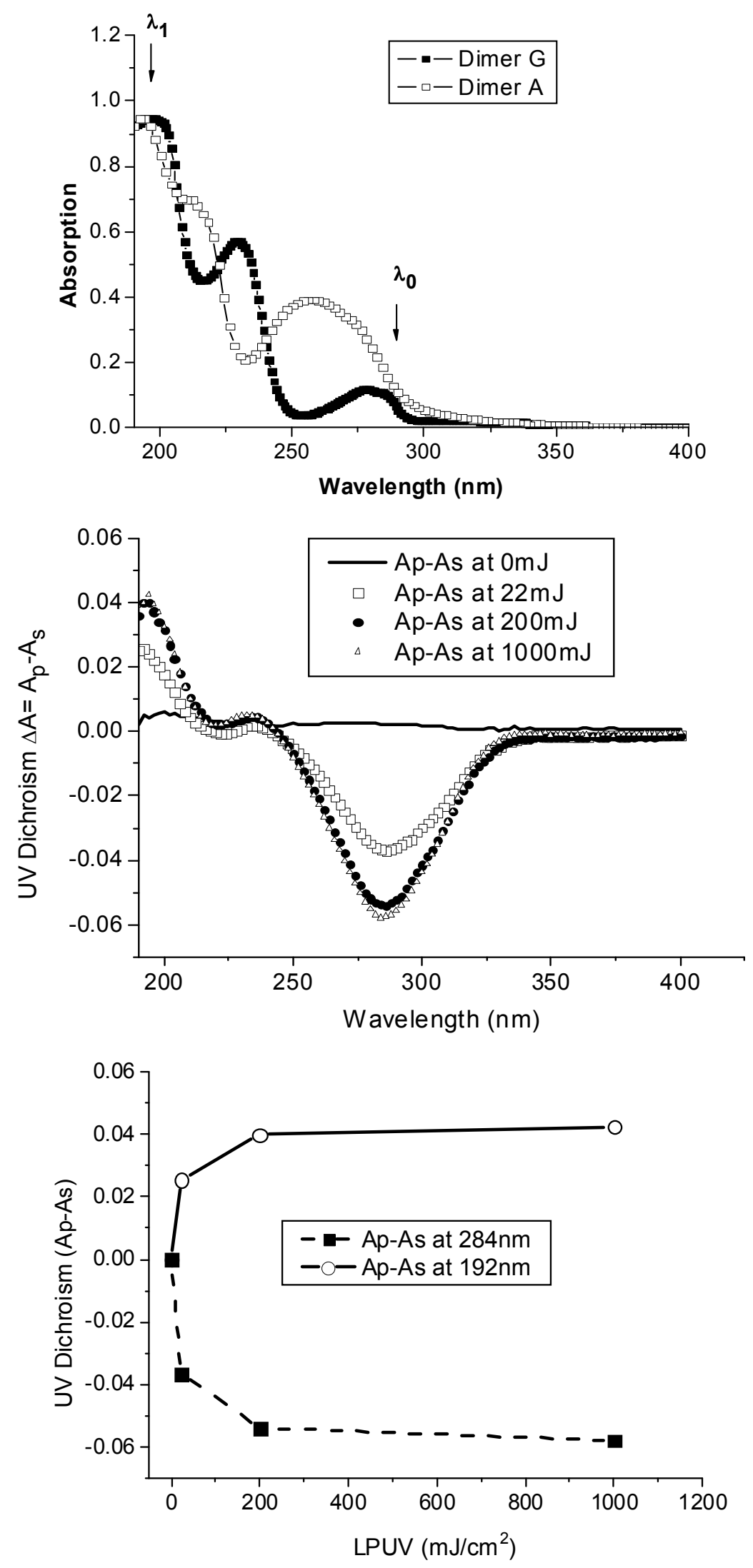

Fig 5d shows the variation of UV absorption dichroism at 284 and $192 \mathrm{~nm}$ as function of LPUV exposure doses. For typical ROP-VA material, strong dichroism and therefore large anisotropies are observed already at low LPUV exposure doses. 


\section{Alignment Mechanism and Performances of LCMO-VA technology}

In the previous sections, we have shown that cross-linking and cyclobutane derivatives are formed in thin films of ROP-VA starting from low LPUV exposure doses. We have also shown that this linear photopolymerisation of ROP-VA layers is accompanied with pronounced optical changes and anisotropies which are known to force liquid crystals to align homogeneously. Based on these results, we suggest in this paragraph possible mechanism which may individually or collectively lead to homogeneous LC photo-alignment.

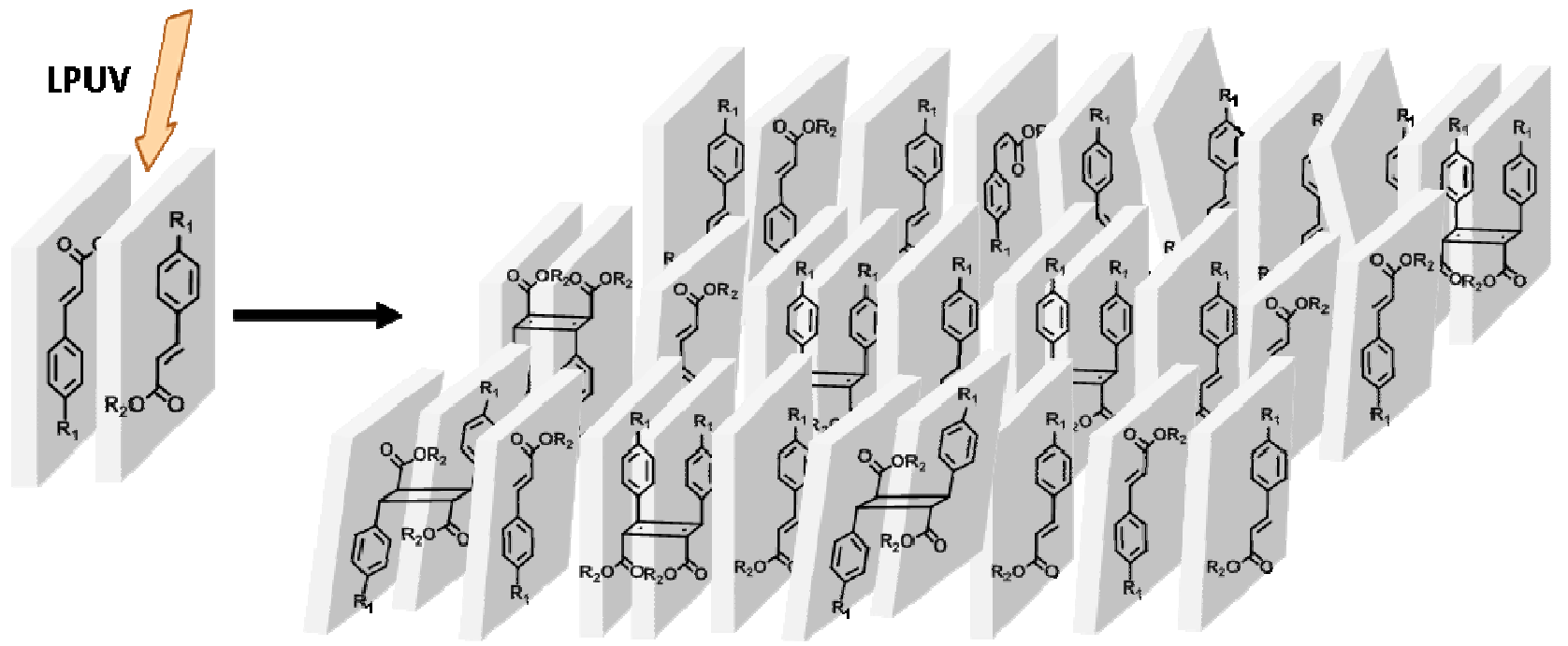

Fig. 6. Schematic of possible resulting molecular configuration and dimerisation occurring in linearly photo-polymerised ROP-VA films.

The results on photo-crosslinking of ROP-VA films with LPUV light can be interpreted at the mesoscopic level as follow: i) LCMO-VA LPUV exposure leads to a preferred depletion (or consumption) of cinnamic side chain molecules along polarised light direction and having maximum absorption, ii) This decreases the effective amount of cinnamate molecules parallel to LPUV and hence increases the effective number of cinnamate molecules perpendicular to LPUV direction and iii) LCMO-VA creates an anisotropic distribution of cyclobutane photo-products with their long axis parallel to LPUV direction. According to this model, the negative dichroism at 284 $\mathrm{nm}$ can be explained according to ii) and the positive dichroism at $192 \mathrm{~nm}$ is related to the alignment and organisation of the resulting cyclic photoproducts along polarisation direction. It is clear that cyclo-butane sub-unit has an isotropic distribution and can not contribute to the anisotropy, however, the resulting photoproducts bearing aromatic moities, are anisotropic. Therefore one can clearly conclude that for our typical ROP-VA the cyclobutane photoderivatives align parallel to the polarisation direction of the linearly polarised UV light, which in turn induce alignment of liquid crystals parallel to linear polarisation direction.

On the microscopic level, we are investigating and probing cinnamate and phenyl groups orientation of ROP-VA alignment layers using other analytical tools such as NEXAFS [19]. At this stage the results indicate that, prior to LPUV exposure, side chain chromophores of ROP-VA are aligned perpendicular to $(\mathrm{x}, \mathrm{y})$ plane with phenyl groups randomly aligned around the normal as schematically shown in Fig. 7a. This is also in agreement with lack of in in-plane anisotropy discussed above. However, after LPUV exposure, microscopic rearrangement occurs and, at least some of, phenyl groups are reoriented in the direction perpendicular to LPUV i.e. to plane (x,z) [19]. It is obvious that Fig. $7 \mathrm{~b}$ is a just a simplified scheme for illustration purpose since, it is not necessary that all side chain chromophores rearrange, only some amount can be sufficient to induce the required alignment and pretilt homogeneity. More detailed investigations are still in progress to clarify the microscopic picture [19]. 
(a)

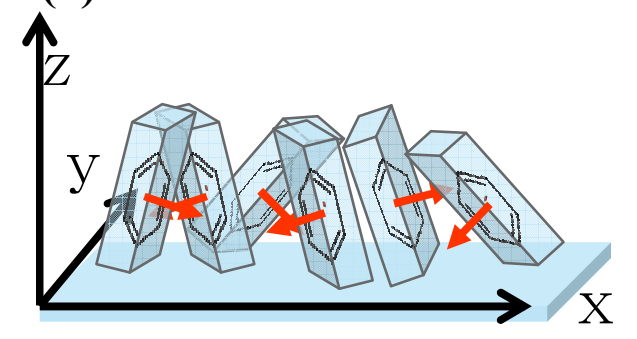

(b)

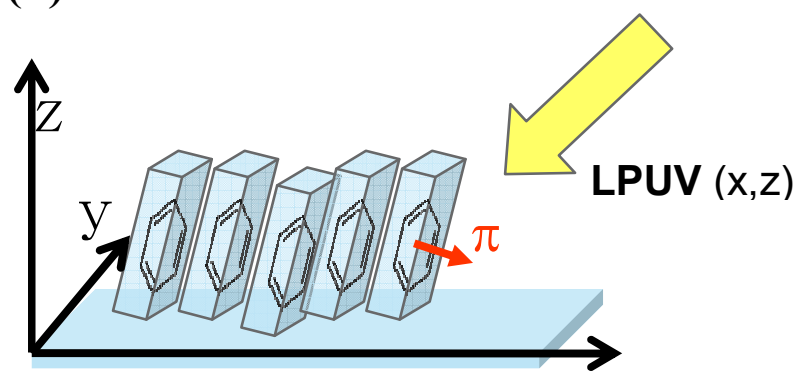

Fig. 7. Schematic representation of phenyl rings of side chain chromophores before LPUV exposure (left) and after LPUV exposure in (x,z) plane (right). The arrows indicate the direction of $\pi$-orbitals of phenyl groups.

Fig. 8a shows that LC is vertically oriented (homeotrope) even without LPUV exposure. The measured tilt angle of $90^{\circ}$ is in line with the normal and random in-plane orientation of chromophores as explained in Fig. 7a. However, application of external voltage induces schlieren defects as shown in Figs $8 \mathrm{~b}$ and $8 \mathrm{c}$, hence, this alignment is not homogeneous and not stable. After LPUV exposure and, hence, cross-linking and subsequent rearrangement of side chain chromophores, liquid crystals are aligned vertically (Fig. 8d) with a tuned pretilt in the range of 87$89^{\circ}$ as required for VA-LCDs. Because of high-sensitivity and cross-linkability of our ROP-VA materials, very homogenous and stable alignment is achieved already at low LPUV exposure doses. External changes such as voltage (Fig. 8e-f) or temperature have no substantial effect on the alignment quality and pretilt angles.
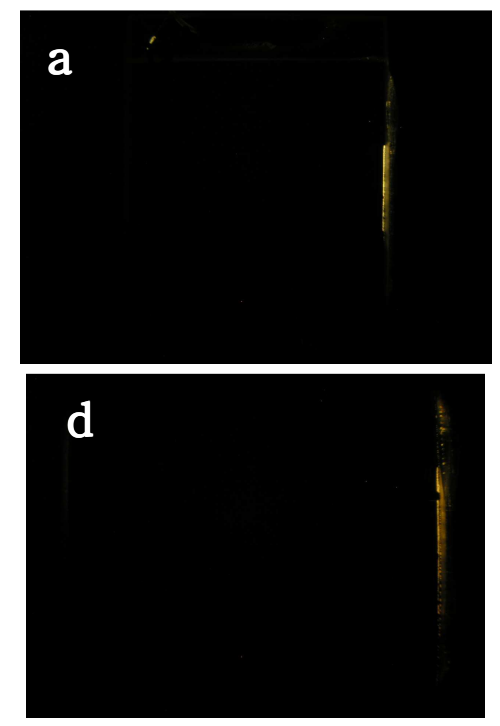
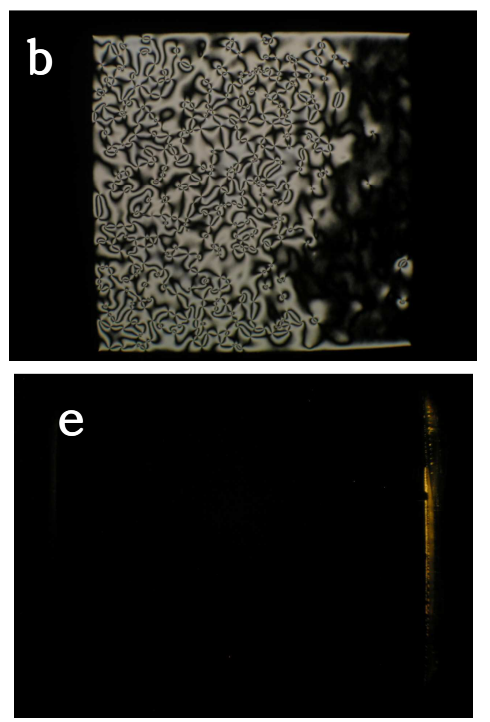
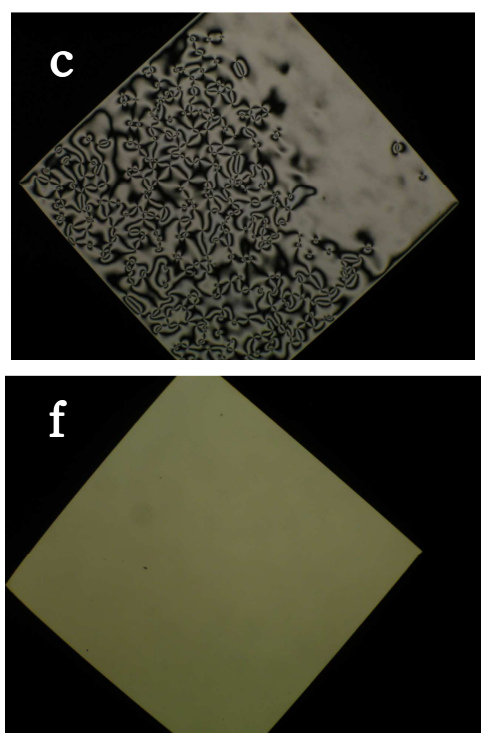

Fig. 8. Comparison of alignment homogeneity of LCMO exposed and non-exposed VA cells made with typical ROP-VA material; a) cell without LPUV exposure and without applied voltage (off-state @ 0V), b-c) cell without LPUV exposure and with applied voltage (on-state @ 5V), d) cell with LPUV exposure and without applied voltage (off-state @ 0V), e-f) cell with LPUV exposure and with applied voltage (on-state @ 5V). All observations are made between cross-polarisers; the angle between polariser and cell edge (respectively LPUV direction) is $90^{\circ}$ for $\mathrm{a}, \mathrm{b}, \mathrm{d}, \mathrm{e}$ and $45^{\circ}$ for $\mathrm{c}, \mathrm{f}$.

Because of the generation of uniform surface with stable alignment and pretilt without fluctuations over large areas, LCMO-VA technology has been successfully applied to Gen 10 factory as the basis technology for the state of the art Ultraviolet induced multi-domain Vertical Alignment (UV2 A) production technology [8, 9]. LCMO-VA allows manufacturing of advanced new generation LCD-TV panels with reduced manufacturing costs and low energy consumption $[8$, 9]. The transmittance, contrast ratio and response time of LCD-TV are drastically improved compared to conventional MVA [8]. 


\section{Summary}

We have shown intrinsic photo-crosslinking of cinnamate groups occurs at low LPUV doses and, allow optically stable alignment and tilt angles on large areas without fluctuations. Direct proof for the occurrence of cycloaddition was obtained by hydrolyzing the cross-linked films of ROP-VA and analysing for cyclobutane derivatives in the hydrolysate. Among other photo-alignment methods, our LCMO technology shows superior performances combining uniform generation of well defined tilt angles over large area without fluctuations, fast and reproducible manufacturing process and high stability of the alignment and tilt angles. This render Rolic ${ }^{\circledR}$ LCMO the fundamental photo alignment technology for the today's advanced displays and optical devices.

Our R\&D efforts are progressing and we believe that Rolic ${ }^{\circledR}$ LCMO technologies will significantly evolve for the development of the next generation displays and non-displays applications. Besides high performances and lower costs, Rolic R\&D activities also consider the global environment issue that has recently become more significant. Rolic is not considering only the reduction of power consumption of LCD and non-LCD devices, but also developing new advanced materials with high sensitivity, still high stability, to reduce production time and, hence, global manufacturing energy and costs.

\section{Aknowledgments}

We wish to thank Dr. Hubert Seiberle for useful discussions.

\section{References}

[1] M. Schadt, K. Schmitt, V. Kozinkov and V. Chigrinov: Jpn. J. Appl. Phys. Vol. 31(1992), p. 2155.

[2] M. Schadt and H. Seiberle: SID 97 DIGEST, 397 (1997).

[3] M. Schadt, H. Seiberle and A. Schuster: Nature Vol. 381(1996), p. 212.

[4] M. Schadt, H. Seiberle, A. Schuster and S. Kelly: Jpn. J. Appl. Phys, Vol. 34 (1995), p. 3240.

[5] H. Seiberle and M. Schadt: Journal of the SID 8/1, 67 (2000).

[6] M. Ibn-Elhaj and M. Schadt: Nature, Vol. 410 (2001), p. 796.

[7] H. Seiberle, T. Bachels, C. Benecke and M. Ibn-Elhaj: IEICE Trans. Electron. Vol. E90-C (2007), p. 2088.

[8] K. Miyachi, K. Kobayashi, Y. Yamada and S. Mizushima: SID 10 DIGEST, 579 (2010)

[9] Y. Yamada, T. Sakurai and K. Miyachi, SID-ME Spring Meeting (2011).

[10]M. Eich and J. H. Wendorff, Macromol. Chem. Rapid Comm. 8, 467 (1987)

[11]T. Todorov, L. Nikolova and N. Tomova, Appl. Optics 23, 4309 (1984)

[12]K. Ichimura, Y. Suzuki, T. Seki, A. Hosoki and K. Aoki, Langmuir 4, 1214 (1988).

[13]W M Gibbons, P J Shannon, S T Sun and B J Swetlin, Nature 351, 49 (1991)

[14]K. Limura, J. Kusano, S. Kobayashi, Y. Aoyagi and T. Sugano, Jap. J. Appl. Phys. 3, L93 (1993)

[15]M. Hasegawa and Y. Taira, J. Photopoly. Sci. Technol. 8 (1995), p. 241.

[16]J. Lu, S.V. Deshpande, E. Gulari, J. Kanicki and W. L. Warren, J. Appl. Phys. 80 (1996), p. 5028.

[17]P. G Egerton., E.Pitts and A. Reiser, Macromolecules 14 (1981), p. 95.

[18]E. Pretsch, T. Clerc, J. Seibl, W. Simon and K. Bienmann: Springer-verlag, Berlin Heidelberg, 2nd edition, 1989

[19]Preferred in-plane and out-of-plane orientation of $\pi$-orbitals phenyl groups revealed using surface-sensitive and polarisation dependent Near-edge X-ray Absorption Fine Structure (NEXAFS); to be published.

[20]H. Seiberle, K. Schmitt and M. Schadt: EuroDisplay 99 Proceedings, 121 (1999). 